\title{
Compensatory saccades made to remembered targets following orbital displacement by electrically stimulating the dorsomedial frontal cortex or frontal eye fields of primates
}

\author{
E.J. Tehovnik *, M.A. Sommer \\ Department of Brain and Cognitive Sciences, Massachusetts Institute of Technology, E25-634. Cambridge, MA (22139, USA
}

Accepted 16 April 1996

\begin{abstract}
If the eye-position signal during visually-evoked saccades is dependent on the dorsomedial frontal cortex (DMFC), one would expect that saccades generated to briefly presented visual targets would be disrupted after displacement of the eyes via electrical stimulation of this cortical area. Compared are compensatory saccades evoked to brief targets following stimulation of the DMFC and frontal eye fields (FEF). Compensatory saccades produced to brief targets following perturbation via the DMFC were not affected. Accordingly, electrical stimulation of the DMFC does not disrupt the eye-position signal during the execution of visually-evoked saccades.
\end{abstract}

Ke'vord:: Saccade: Remembered target; Dorsomedial frontal cortex: Frontal eye field; Electrical stimulation: Rhesus monkey

Monkeys are able to generate saccades to remembered target positions after their eyes are displaced by electrical stimulation of either the frontal eye fields (FEF) or superior colliculi, but not after being displaced by stimulation of the trochlear or abducens nucleus or by stimulation of the paramedian pontine reticular formation or fastigial nuclei $[6,8,10,13,14]$. This suggests that electrical stimulation of the trochlear or abducens nuclei or paramedian pontine reticular formation or fastigial nuclei disrupts the eye-position signal during visually-evoked saccades.

Models of the brainstem saccade generator use as an input a signal representing 'desired' eye position in craniotopic coordinates [9]. We have suggested that the dorsomedial frontal cortex (DMFC), which innervates the brainstem [4,12], might hold such an eye-position signal for generating visually-evoked saccades [5]. The activity of units within the DMFC is modulated by changes in eye position [1,5,11]. Over $60 \%$ of the units have activity related to visual fixation, and of these over $40 \%$ become tonically active before the eyes arrive on target [5]. Electrical stimulation of the DMFC drives the eye to a given craniotopic location, a termination zone, and once within

\footnotetext{
"Corresponding author. Fax: + 1 (617) 253-8943; E-mail: tehovnik (a) wect mit.edu
}

this termination zone further stimulation holds the eyes at that position and inhibits all visually-elicited saccades [16.17]. Termination zones are topographically represented in the DMFC, and these zones are anchored to the head $[5,16,17]$. If the eye-position signal for visually-evoked saccades is dependent on the DMFC. one would expect that saccades generated to a remembered target position would be disrupted after perturbation via DMFC stimulation. Compared are corrective saccades evoked to briefly presented visual targets following stimulation of either the DMFC or FEF.

Two adult rhesus monkeys (Macaca mulatta), I and L were used. Details pertaining to surgery, data collection, and electrical stimulation can be found in Tehovnik and Lee [16]. The monkeys were water deprived overnight before each day of experimental testing. After testing, they were allowed to drink to satiation before being returned to the vivarium. For each monkey, a recording chamber was implanted over the DMFC, and another chamber was implanted over the left FEF.

During behavioural testing, a monkey faced a 40 degree wide by 30 degree high LED board positioned $108 \mathrm{~cm}$ away with head fixed. The animal had to fixate an LED with diameter of 0.3 degrees of visual angle for $500 \mathrm{~ms}$. During fixation, a monkey had to keep his eyes within a $5 \times 5$ degree window; otherwise the trial was terminated. After the fixation spot was turned off, a second LED was 
flashed for $50 \mathrm{~ms}$. The monkey was required to produce a saccade to the flashed LED; the eyes had to enter a $10 \times 10$ degree target window in order to obtain a drop of apple juice. At least three target positions were tested. From trial to trial, the targets were presented in random order and they were typically 10 degrees from the fixation spot. On 15\% of trials, a target was not flashed. Here the monkey was required to remain fixated for $100 \mathrm{~ms}$ to obtain the reward.

After a monkey was proficient at the task (error rate < $5 \%$ ), either the DMFC or FEF was electrically stimulated immediately after target extinction. Stimulation was deliv-

\section{Left}

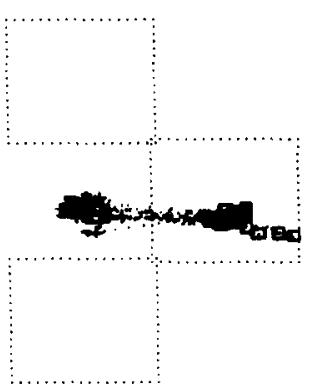

B
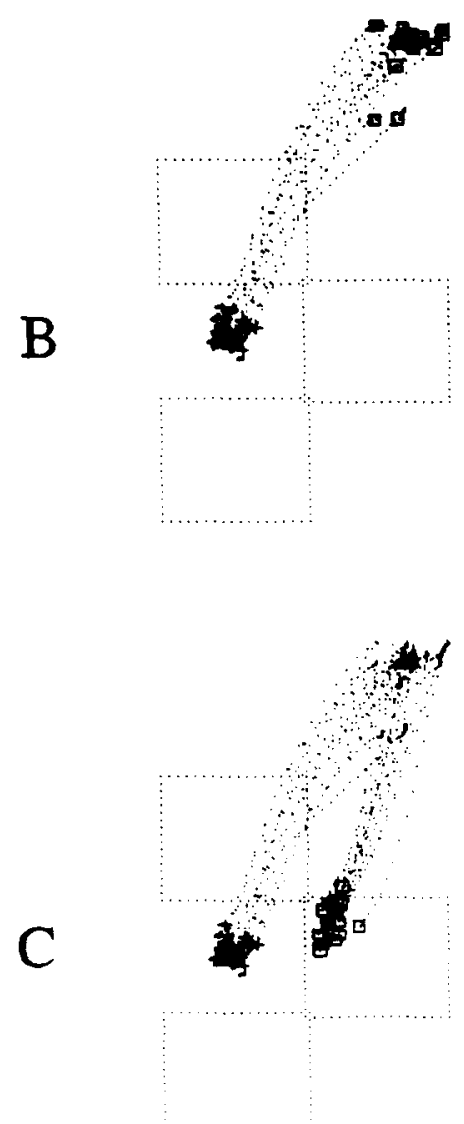
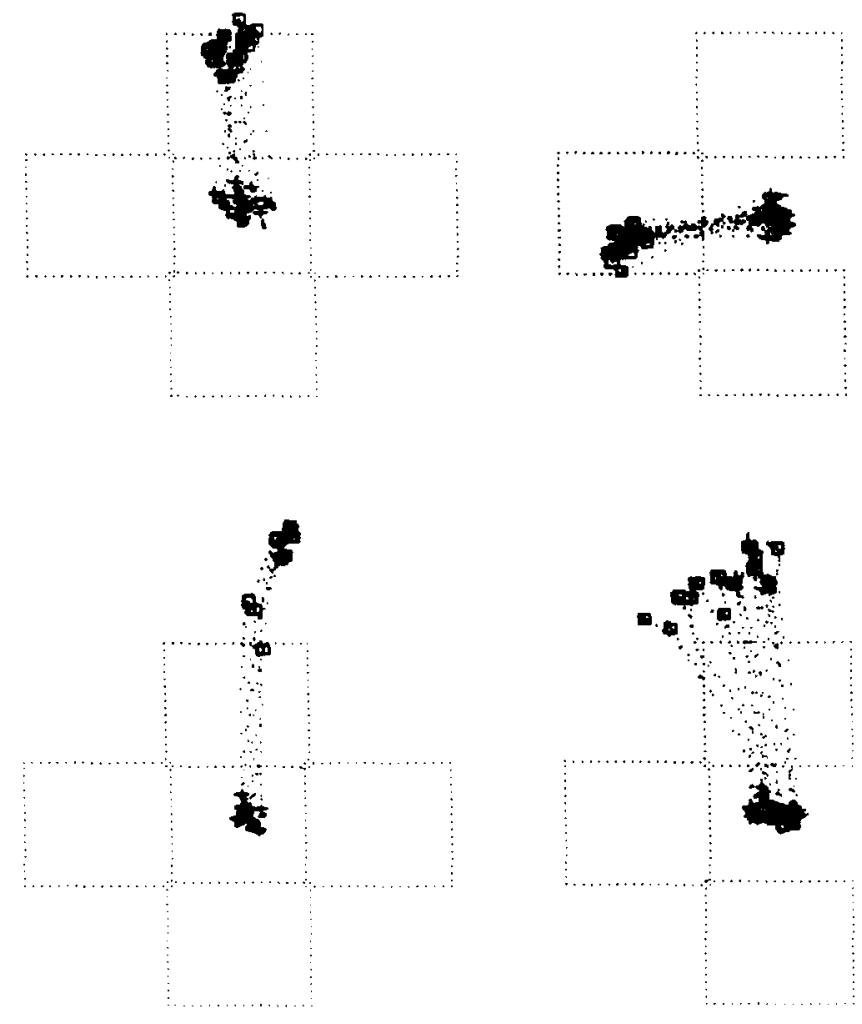

Right
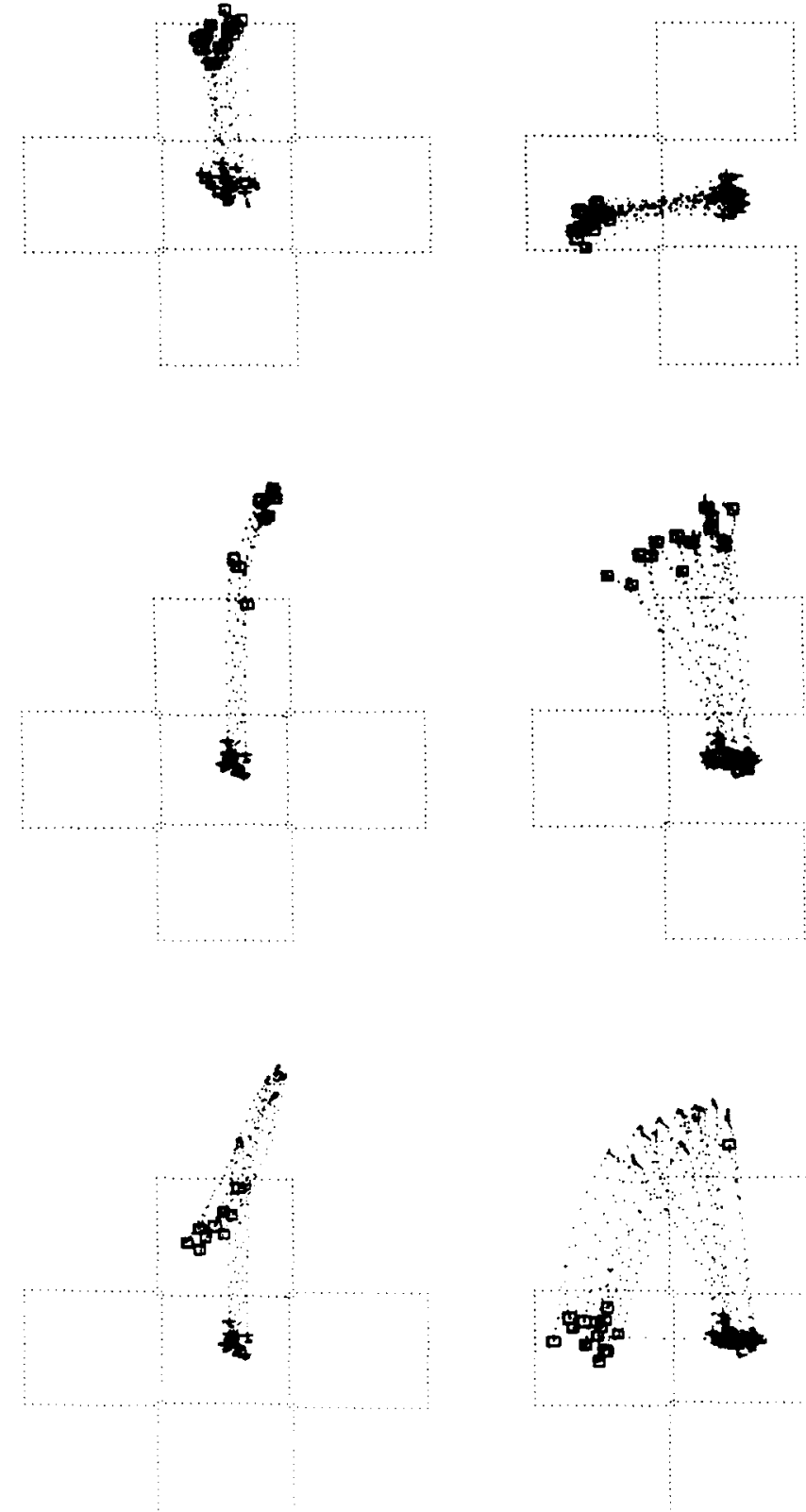

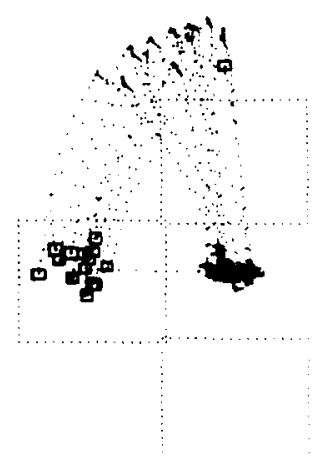

Fig. 1. Saccades made to brief targets (A), stimulation-evoked saccades (B), and compensatory saccades to the targets after perturbation via stimulation (C) are shown for stimulation of a site within the DMFC. Within a plate, each square represents a window about a target. Targets were 10 degrees from the fixation spot. Fixations are marked by crosses and saccadic terminations are marked by small squares. The dots between a cross and a square represent the trajectory of one saccade (A and B) or two saccades (C) for a single trial. Fixations were made to fixation spots in left (Left). center (Center), or right (Right) craniotopic space. The left and right fixation spots were 40 degrees apart along the horizontal axis, and they were each 20 degrees from the center fixation spot. Stimulation used to perturb the eyes consisted of $200-\mathrm{ms}$ trains of $400 \mu \mathrm{A}$ pulses (at a $0.1 \mathrm{~ms}$ pulse duration) delivered at $150 \mathrm{~Hz}$. Stimulation began immediately after the target was extinguished. 
ered on 30 to $50 \%$ of the trials. To eliminate all extraneous visual cues while a monkey produced saccades to a flashed target, the house light was extinguished at target presentation and remained off until the start of the next trial [10].

For electrical stimulation, glass-coated platinum-iridium electrodes with an impedance of 0.1 to $1.0 \mathrm{M} \Omega$ at $1 \mathrm{kHz}$ were constructed. Constant-current biphasic pulses were delivered to the brain tissue using a Grass S88 stimulator attached to a pair of constant-current, stimulus isolation units (Grass PSIU6B). A total of 11 and 20 penetrations were made into the DMFC and FEF, respectively, of the two monkeys. The FEF and DMFC were defined physiologically $[2,5,16]$. The location of the electrodes penetrations was determined by noting the location of a recording chamber relative to the arcuate sulcus and cortical midline during surgery, and by noting the electrode coordinates. Both monkeys are being used for further study.

A PDP $11 / 73$ computer controlled the presentation of visual stimuli, the delivery of electrical stimulation, the assessment of correct saccadic responses using target windows, the storage of task-related events, and the collection of eye movements. An eye movement (sampled at $333 \mathrm{~Hz}$ ) had to achieve an instantaneous velocity of at least 100 $\mathrm{deg} / \mathrm{s}$ to qualify as a saccade.

Monkeys made saccades to a flashed target from one of three fixation spots located in left, center, or right portions of craniotopic space (Fig. 1A). Saccades were evoked by stimulation of the DMFC following fixations in left, center, or right craniotopic space (Fig. 1B). After the end of such stimulation, saccades put the eyes on target (Fig. 1C). Similar results were seen with targets flashed elsewhere and with all sites tested in the DMFC. Thus compensatory saccades were produced following stimulation of the DMFC regardless of the location of the fixation spot, and the dispersion of these saccades did not differ appreciably from those evoked to the flashed targets in the absence of stimulation

Using the same conditions of electrical stimulation, compensatory saccades were produced following stimulation of the FEF (Fig. 2). Saccades were generated to a flashed target from a fixation spot located in left craniotopic space (Fig. 2A), and saccades were elicited from the fixation spot by stimulation of the FEF (Fig. 2B). After the end of stimulation, saccades put the eyes on target (Fig. 2C).

The current results indicate that electrical stimulation of the DMFC does not disrupt the eye-position signal during visually-evoked saccades. To date, the eye-position signal has been disrupted via stimulation of oculomotor or fastigial nuclei or sites within the paramedian pontine reticular formation $[8,10,13,14]$, but not disrupted via stimulation of the superior colliculus, FEF, or DMFC (current study, $[6.10,13])$. Thus the eye-position signal is dependent on structures residing subjacent to the colliculus and is not dependent on the DMFC.

The precise role of the DMFC in oculomotor control is

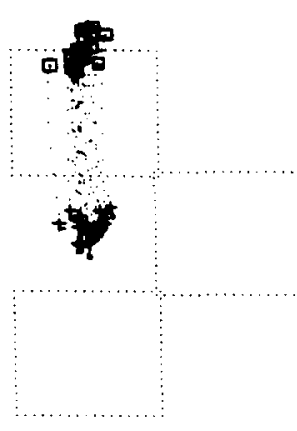

A
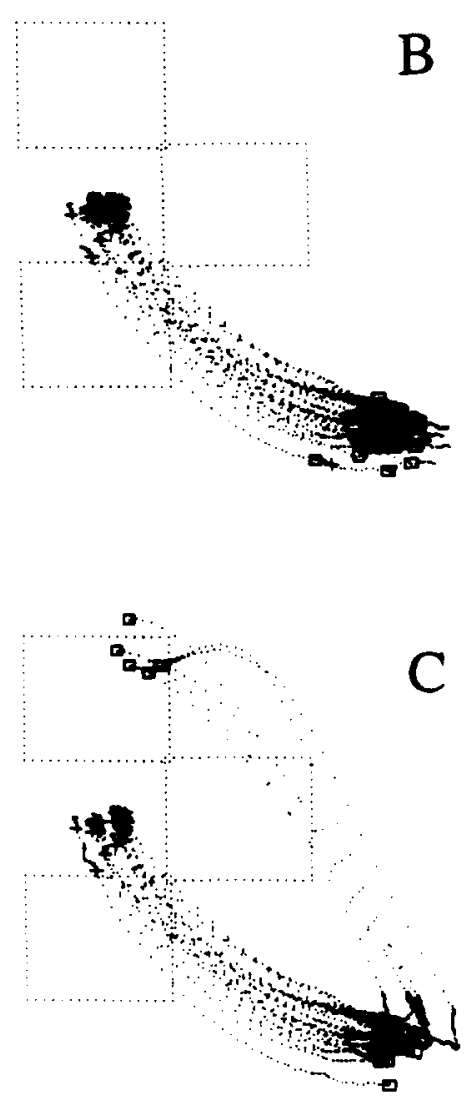

Fig. 2. Saccades to a brief target (A), stimulation evoked saccades (B). and compensatory saccades to the target after perturbation following stimulation (C) are shown for stimulation of a site within the FEF. Initial fixations were made to a fixation spot in left craniotopic space. See Fig. 1 for other details.

unclear. Unlike the FEF and superior colliculi, the DMFC has a neuronal representation of the skeletal musculature [15]. Furthermore, it has been implicated in learning oculomotor and skeletomotor tasks [3,7]. The topographic representation of eye position in the DMFC $[5,16,17]$ might be involved in establishing a linkage between the various motor systems during the learning of motor tasks. This idea is currently under investigation.

In conclusion, compensatory saccades are evoked fol- 
lowing perturbation of the eyes via stimulation of the DMFC, which concurs with results in the superior colliculus and FEF. Thus electrical stimulation of the DMFC does not disrupt the eye-position signal during visually-evoked saccades.

\section{Acknowledgements}

This work was supported by NIH EY08502 to P.H. Schiller. We would like to thank Dr. Peter Schiller for taking the time to review this work.

\section{References}

[1] Bon, L. and Lucchetti, C., The dorsomedial frontal cortex of the macaca monkey: fixation and saccade-related activity, Exp. Brain Res., 89 (1992) 571-580.

[2] Bruce, C.J., Goldberg, M.E., Bushnell, M.C. and Stanton. G.B., Primate frontal eye fields. II. Physiological and anatomical correlates of electrically evoked eye movements, J. Neurophysiol., 54 (1985) 714-734

[3] Chen, L.C. and Wise, S.P., Neuronal activity in the supplementary eye field during acquisition of conditional associations, J. Neurophysiol., 73 (1995) 1101-1120.

[4] Huerta, M.F. and Kaas, J.H., Supplementary eye fields as defined by intracortical microstimulation: connections in macaque, J. Comp. Neurol, 293 (1990) 299-330.

[5] Lee, K.-M. and Tehovnik, E.J., Topographic distribution of fixation-related units in the dorsomedial frontal cortex of the rhesus monkey, Eur. J. Neurosci., 7 (1995) 1005-1011.

[6] Mays, L.E. and Sparks, D.L., Saccades are spatially, not retinocentrically, coded, Science, 208 (1980) 1163-1165.
[7] Mushiake, H., Inase, M. and Tanji, J., Neuronal activity in the primate premotor, supplementary and precentral motor cortex during visually guided and internally determined sequential movements, $J$. Neurophysiol, 66 (1991) 705-718.

[8] Noda, H.. Shinji, M. and Warabi, T., Effects of fastigial stimulation upon visually-directed saccades in macaque monkeys. Neurosei. Res., 10 (1991) 188-199.

[9] Robinson, D.A., Oculomotor control signals. In G. Lennerstrand and P. Bach-y-Rita (Eds.), Basic Mechanisms of Ocular Motility and their Clinical Implications, Pergamon Press, Oxford, UK, 1975, pp. $337-374$.

[10] Schiller, P.H. and Sandell, J.H., Interactions between visually and electrically elicited saccades before and after superior colliculus and frontal eye field ablations in the rhesus monkey, Exp. Brain Res. 49 (1983) $381-392$.

[11] Schlag, J., Schlag-Rey, M. and Pigarev, I., Supplementary eye field: influence of eye position on neural signals of fixation. Exp. Brain Res. 90) (1992) 302-306.

[12] Shook, B.L., Schlag-Rey, M. and Schlag. J., Primate supplementary eye field. I. Comparative aspects of mesencephalic and pontine connections, J. Comp. Neurol., 301 (1990) 618-642.

[13] Sparks, D.L. and Mays. L.E., Spatial localization of saccade targets. I. Compensation for stimulation-induced perturbations in eye position, J. Neurophysiol. 49 (1983) 45-63.

[14] Sparks. D.L., Mays, L.E. and Porter, J.D., Eye movements induced by pontine stimulation: interaction with visually triggered saccades. J. Neurophysiol., 58 (1987) 300-318.

[15] Tehovnik. E.J., The dorsomedial frontal cortex: eye and forelimb fields, Behar. Brain Res., 67 (1995) 147-163.

[16] Tehovnik. E.J. and Lee, K.-M., The dorsomedial frontal cortex of the rhesus monkey: topographic representation of saccades evoked by electrical stimulation, Exp. Brain Res., 96 (1993) 430-442.

[17] Tehovnik. E.J., Lee, K.-M. and Schiller, P.H., Stimulation-cvoked saccades from the dorsomedial frontal cortex of the rhesus monkey following lesions of the frontal eye fields and superior colliculus, Exp. Brain Res., 98 (1994) 179-190. 